\title{
Muséologies
}

Les cahiers d'études supérieures

\section{Collectionner la performance : un dialogue entre l'artiste et le musée}

\section{Amélie Giguère}

Volume 7, numéro 1, 2014

Le dialogue dans les musées d’art contemporain

URI : https://id.erudit.org/iderudit/1026653ar

DOI : https://doi.org/10.7202/1026653ar

Aller au sommaire du numéro

Éditeur(s)

Association Québécoise de Promotion des Recherches Étudiantes en

Muséologie (AQPREM)

ISSN

1718-5181 (imprimé)

1929-7815 (numérique)

Découvrir la revue

Citer cet article

Giguère, A. (2014). Collectionner la performance : un dialogue entre l'artiste et le musée. Muséologies, 7(1), 169-185. https://doi.org/10.7202/1026653ar

Tous droits réservés (C Association Québécoise de Promotion des Recherches Étudiantes en Muséologie (AQPREM), 2014
Ce document est protégé par la loi sur le droit d'auteur. L'utilisation des services d'Érudit (y compris la reproduction) est assujettie à sa politique d'utilisation que vous pouvez consulter en ligne.

https://apropos.erudit.org/fr/usagers/politique-dutilisation/ 
Article sept

\section{Collectionner la performance:}

un dialogue entre l'artiste et le musée

Amélie Giguère 
Amélie Giguère détient un doctorat en muséologie, médiation, patrimoine de l'Université du Québec à Montréal (UQAM) et de l'Université d'Avignon et des Pays de Vaucluse (2012). Elle a auparavant complété une maîtrise en études des arts (UQAM, 2004) et un baccalauréat en communication (UQAM, 1999). Ses travaux portent sur la conservation et la documentation de l'art contemporain et plus largement sur la muséalisation des œuvres éphémères et performatives. Elle a par ailleurs publié des articles dans plusieurs revues, dont Culture et musées, Esse, Les Cahiers de théâtre Jeu, ETC Montréal, Spirale. Aujourd'hui, elle enseigne l'histoire de l'art et la muséologie au Département d'histoire de l'art de l'UQAM, à l'École multidisciplinaire de l'image de l'Université du Québec en Outaouais (UQO) et au Cégep Marie-Victorin. Précédemment, elle a été journaliste, critique de théâtre et recherchiste à la télévision. amelie.giguere@yahoo.ca 
Au milieu des années 1990, la conservation de l'art moderne et de l'art contemporain devient une préoccupation importante pour de nombreuses institutions muséales. Autour des collections, des groupes et des réseaux de recherche, composés de conservateurs, de restaurateurs, de documentalistes, d'historiens de l'art, mais également d'artistes et de leurs représentants, se forment pour développer des modèles théoriques et des outils pratiques dans le but de traiter de problèmes générés notamment par l'emploi de matériaux fragiles ou par des exigences de mises en exposition complexes ${ }^{1}$.

Au-delà de l'élaboration de modèles et d'outils, ces recherches, qui se poursuivent aujourd'hui, mettent en évidence les transformations de l'institution muséale. Non seulement l'art contemporain exige des professionnels qu'ils acquièrent de nouvelles connaissances et de nouveaux savoir-faire pour assurer la pérennité des œuvres, mais l'entrée de ces œuvres dans les collections tend à redéfinir les théories mêmes de la conservation, ainsi que les responsabilités des professionnels et des artistes appelés à travailler ensemble à l'acquisition, à la conservation et à l'exposition des œuvres.

Rappelons que, dans les théories traditionnelles de la conservation, préserver l'intégrité des objets d'art consiste à préserver leur matérialité, leur forme et leur apparence physique. Dans cette approche de la conservation, l'œuvre d'art est un objet original et authentique, c'està-dire irremplaçable et pourvu d'une valeur

1 Parmi les premières grandes rencontres qui ont eu lieu, mentionnons Responsabilité partagée (Musée des beaux-arts du Canada, Ottawa, 1989), Conservation et restauration des œuvres d'art (École du patrimoine, Paris, 1992), From Marble to Chocolate: the Conservation of Modern Sculpture (Tate Gallery, Londres, 1995), Modern Art: Who Cares? (Netherlands Institute for Cultural Heritage, Foundation for the Conservation of Contemporary Art in the Netherlands, Amsterdam, 1997), Mortality Immortality: the Legacy of 20th-Century Art (The Getty Conservation Institute, Los Angeles, 1998), L'approche des médias variables: la permanence par le changement (Solomon R. Guggenheim Museum et Fondation Langlois, New York, 2001). Depuis le début du XXI' siècle, les rencontres internationales se multiplient.

2 PEARCE, Susan M. On Collecting an Investigation into Collecting in the European Tradition. Londres: Routledge, 1995, p. 14. intrinsèque ${ }^{2}$. Cet objet doit par conséquent être conservé, car il constitue le témoin concret et tangible d'une réalité qui n'est ni concrète ni tangible. Dans cette perspective, ne pas préserver la matérialité de l'œuvre peut provoquer une perte d'informations, détruire ou étioler le lien intangible qui unit les objets au passé et à d'autres réalités.

Dans les théories contemporaines de la conservation, l'intégrité accueille d'autres dimensions: elle est matérielle certes, mais elle est aussi de nature esthétique, historique, voire conceptuelle $^{3}$. Les fonctions et les usages des objets sont également reconnus comme des données signifiantes ${ }^{4}$. Alors qu'il existe, pour les œuvres qui présentent des propriétés matérielles et physiques relativement stables, un état original ou un état idéal qu'il s'agit de préserver, plusieurs œuvres contemporaines sont soumises à d'autres paramètres, se dégradent de manière accélérée ou connaissent des versions multiples. Les propriétés matérielles et physiques de ces œuvres ne peuvent dès lors plus agir comme références. Par conséquent, il devient difficile pour les conservateurs, qui en ont le soin, de déterminer si ces dégradations sont appropriées (et jusqu'à quel point) et si les multiples versions peuvent accuser des variations formelles (et si oui, lesquelles?). En définitive, à l'égard de l'art contemporain, il est fréquent que le respect de l'intégrité conceptuelle de la proposition artistique soit défini comme un principe dominant de conservation.

3 Dans le contexte des nouveaux enjeux, les codes déontologiques des associations de conservateurs identifient de multiples dimensions à l'intégrité. Voir SEASE, Catherine. "Codes of Ethics for Conservation ". International Journal of Cultural Property, vol. 7, no 1, 1998, p. 98-115.

4 CLAVIR Miriam. Preserving What Is Valued: Museums, Conservation, and First Nations. Vancouver: University of British Columbia Press, 2002 ; MUÑOS VINAS, Salvador. Contemporary Theory of Conservation. Oxford et Burlington: Elsevier Butterworth-Heinemann, 2005. 
Respecter l'intégrité d'une œuvre revient généralement à honorer l'intention de l'artiste qui préside à la création de cette dernière ${ }^{5}$. Dans cette perspective, il est admis que le discours de l'artiste sur son travail nourrit l'interprétation des œuvres et guide les mesures entreprises pour assurer leur conservation. Par ailleurs, si le respect de l'intention de l'artiste est un principe qui traverse les différentes théories de la conservation, ce qui est inédit à l'égard de l'art contemporain, c'est la possibilité d'éclairer cette intention en interrogeant directement l'artiste. La parole de l'artiste, vivant au moment où ses œuvres rejoignent les collections muséales, devient une source d'informations incontournable; en effet, comme le rapporte Ijsbrand Hummelen, «The artists were given the opportunity to explain exactly how their choices related to the attainment of their artistic aims. In other words, [their writings acknowledge] the importance of differences in materials and techniques in relation to the artistic intentions ${ }^{6} . "$

La prise en charge de ce discours, à travers des questionnaires ou des entretiens, est au cœur d'une pratique optimale de la conservation. Plus que simplement recueillir de l'information, les professionnels et les artistes engagent un dialogue. Si des sources textuelles et iconographiques sont prises en compte dans le travail de conservation de l'art contemporain, le dialogue est certainement un canal largement exploité.

Dans cet article, nous analysons ce dialogue pour comprendre comment se négocie la conservation de l'art contemporain et, de

5 Rappelons toutefois que la question de l'intention, de sa définition, de la possibilité de l'identifier sur la base des choix plastiques du créateur et sur son rôle dans l'interprétation du travail artistique n'apparaît pas avec les problèmes de conservation de l'art contemporain et n'est pas sans controverse. Pour une présentation des différents enjeux et débats autour de l'intention, voir DYKSTRA, Steven W. "The Artist's Intention and the Intentional Fallacy in Fine Arts Conservation ". Journal of the Institute for Conservation, vol. 35, no 3, 1996, p. 197-218. 6 HUMMELEN, Ijsbrand. "Conservation Strategies for Modern and Contemporary Art: Recent Developments in the Netherlands ", Cr, no 3, 2005, p. 22. <www.inside-installations.org/OCMT/mydocs/HUMMELEN\%20Conservation $\% 20$ Strategies $\% 20$ for\%20Modern $\% 20$ and $\% 20$ Contemporary\%20Art_1.pdf> (consulté le 1er février 2013). Voir aussi LAURENSON, Pip. «The Preservation of Installations $"$. In. $<w w w$.inside-installations.org $>$, sous la direction de l'Instituut Collectie Nederland, p. 43. <www.incca.org/files/ manière plus précise, la conservation de la performance, qui est certainement l'une des pratiques les plus radicales du point de vue du rapport qu'elle entretient avec le musée. Nous observons que le dialogue intervient à deux moments décisifs. En effet, c'est à travers celui-ci que les artistes et les professionnels déterminent la forme que prendra la "performance » dans la collection muséale. Et c'est aussi en partie à travers des échanges que sont éclairés le statut des documents issus ou associés à la performance, de même que les rapports qu'ils entretiennent avec cette dernière. Nous entendons « dialogue " a priori selon son " sens restreint » issu du grec dialogos, qui signifie simplement " entretien, discussion ». Suivant l'idée généralement admise que le "dialogue idéal " est un " échange constructif " entre deux personnes, nous retenons que celui-ci existe dans un "mouvement dialectique impliquant tout à la fois identité et différence ${ }^{7}$. Nous supposons qu'il est d'abord oral, mais nous acceptons qu'il soit écrit, qu'il prenne la forme d'échanges électroniques, par exemple. Et par "performance ", nous entendons le large spectre des actions contemporaines ou actuelles qui possèdent une durée de réalisation et pour lesquelles les gestes d'exécution de l'artiste ou des interprètes ont une valeur artistique intrinsèque. Nous envisageons la diversité des pratiques artistiques contemporaines ou actuelles du domaine des arts visuels qui empruntent aux traditions plasticiennes, théâtrales, littéraires ou musicales ${ }^{8}$.

pdf/projects_archive/2007_Inside_Installations_booklet.pdf> (consulté le 1er février 2013).

7 CHARAUDEAU, Patrick et Dominique MAINGUE-

NEAU. «Dialogue ». Dictionnaire d'analyse du discours. Paris: Seuil, 2002, p. 178-180.

8 L'élargissement du champ de la performance rend périlleux l'exercice de définition. Nous reprenons à notre compte la proposition d'Éric Mangion, élaborée à l'occasion d'une exposition sur le thème de la performance et de ses objets, présentée à la Villa Arson en 2008, qui a convenu que la performance est un terme générique qui englobe toutes les typologies d'actions du domaine des arts visuels, comme le happening, l'event, le body art ou les interventions plus conceptuelles. MANGION, Éric. « La finition fétichisante de la performance, entretien réalisé par Raphaëlle Giancreco ". In. MANGION, Éric et Marie de BRUGEROLLE (dir.). Ne pas jouer avec des choses mortes. Dijon: Les presses du réel; Nice: Villa Arson, p. 13. 
Trois parties composent cet article. Dans un premier temps, nous évoquons les enjeux particuliers de la collection et de la conservation de la performance. Puis, à travers l'étude de l'entrée récente des performances d'Orlan (MesuRage du Centre Georges Pompidou, créée en 1977) et de Claudie Gagnon (Les Époux Arnolfini, créée en 2008) dans les collections respectives du Musée national d'art moderne (MNAM) du Centre Pompidou et du Musée national des beaux-arts du Québec (MNBAQ), nous observons comment se négocie la transposition de propositions performatives en des " objets de musée " ou, du moins, en des propositions inédites qui tendent à " retrouver " les attributs de l'objet de musée. Enfin, nous interrogeons la mise en lumière du statut des objets et des documents issus de performances dans la conservation optimale de ce travail artistique singulier.

\section{La consultation des dossiers documentaires} de ces deux œuvres, qui conservent des traces des échanges et des négociations (des notes, des communications électroniques, des listes d'objets, de noms, de titres préliminaires, etc.), de même que l'examen de leurs fiches descriptives abrégées et détaillées, nous permettent d'interroger ces deux cas. Des entretiens réalisés auprès des professionnels ou des artistes complètent la collecte des données. Dans le cas de la performance d'Orlan, c'est une discussion avec la documentaliste des collections du MNAM et un échange électronique avec la conservatrice responsable de l'acquisition de la pièce qui nous apportent les informations pertinentes ${ }^{9}$. Dans le cas de la performance de Claudie Gagnon, ce sont des entretiens avec l'artiste et avec la conservatrice de l'art actuel du MNBAQ de même que la participation à une discussion à trois qui constituent nos sources principales ${ }^{10}$.

9 La discussion avec la documentaliste Evelyne Pomey est réalisée au Centre Pompidou, le 5 octobre 2009. L'échange électronique avec la conservatrice Sophie Duplaix a lieu le 23 octobre 2009.

10 Trois entretiens enregistrés avec la conservatrice Nathalie de Blois sont réalisés (les 5 mars et 20 juillet 2009, et le 14 septembre 2011). La discussion enregistrée entre l'artiste et la conservatrice à laquelle nous participons a lieu au MNBAQ, le 25 mars 2009. Enfin, nous avons un entretien téléphonique avec l'artiste le 10 septembre 2011.
Intégrer la performance dans les collections
muséales: une entreprise controversée

L'entrée de la performance dans les collections muséales demeure une entreprise controversée. D'un point de vue pragmatique, la performance peut difficilement être conservée, car elle répond à un mode d'existence diamétralement opposé à celui des objets de musée traditionnels autour desquels les activités muséales se sont développées. Ces objets traditionnels sont en effet dotés d'une matérialité relativement stable qui leur permet d'être entreposés dans une réserve, restaurés ou présentés sur une cimaise, sur un socle ou directement sur le sol. S’il est vrai que depuis deux décennies le musée a développé des outils pour accueillir des formes contemporaines plus complexes, comme l'installation, l'œuvre conceptuelle ou à composantes électroniques, la performance, parce qu'elle se structure autour de la personne ou du corps d'un artiste en action, suscite des difficultés inédites et de nouvelles questions.

D'un point de vue idéologique, la performance est traditionnellement définie comme une pratique critique à l'endroit des grandes institutions de l'art et du marché. À l'instar de l'art conceptuel, la performance participe du phénomène de la " dématérialisation de l'art ", défendu par Lucy R. Lippard à la fin des années 1960. Cette approche de la création annonce l'obsolescence de l'objet dans l'art et revendique la reconnaissance d'un " art comme idée " et d'un "art comme action " ${ }^{11}$. Suivant ces discours, l'entrée de la performance dans les collections apparaît nécessairement paradoxale et semble relever d'une forme de récupération du musée, ou de la pratique elle-même. Quant à l'approche documentaire de l'intégration muséale de la performance, elle apparaît aux yeux de plusieurs observateurs comme un

11 LIPPARD, Lucy R. et John CHANDLER. "The Dematerialization of Art ». Art International, vol. 12, no 2, 1968 , p. 31. 
compromis peu satisfaisant, car elle n'exploite que le régime second de la trace ou du "produit dérivé ${ }^{12}$.

Bien qu'elles conservent leur pertinence, ces deux observations doivent être nuancées, notamment par d'autres approches de la performance. Nombreux sont les artistes qui accordent une valeur historique, esthétique ou artistique aux objets qui lui sont associés. Plusieurs récupèrent, réorganisent ou produisent des objets, des photographies ou des vidéos dans le but de témoigner, de prolonger et de réinterpréter leur travail performatif. Gina Pane, par exemple, accordait un grand soin à la réalisation des " constats photographiques " de ses actions, qu'elle définissait comme partie intégrante de son travail de performance. Conscients du pouvoir des images et de la facilité avec laquelle elles circulent, de jeunes artistes, comme Thierry Marceau, développent parallèlement et avec autant de considération le travail performatif et celui de sa documentation photographique. Tout à fait différemment, d'autres artistes développent des systèmes qui leur permettent de reprendre leurs performances, que celles-ci aient été pensées au départ pour être reprises ou non. Empruntant l'économie des actions du mouvement Fluxus, structurées autour de partitions destinées à être réinterprétées, les performances d'Esther Ferrer sont conçues pour être rejouées dans des contextes variables. Enfin, il y a des artistes qui réactualisent leurs performances ou celles d'autres artistes en se mettant en scène ou en recourant à des interprètes.

12 Pour une présentation plus complète du débat et des auteurs, voir GIGUÈRE, Amélie. Art contemporain et documentation: la muséalisation d'un corpus de pièces éphémères de type performance. Thèse de doctorat en muséologie, médiation, patrimoine, Université du Québec à Montréal et Université d'Avignon et des Pays de Vaucluse, 2012, p. 52-57.

13 PHELAN, Peggy. Unmarked: the Politics of Performance. Londres et New York: Routledge, 1993; JONES, Amelia.

" "Presence" in Absentia: Experiencing Performance as Documentation ". Art Journal, vol. 56, no 4, 1997, p. 11-18; AUSLANDER, Philip. "The Performativity of Performance Documentation ", Performing Arts Journal, no 84, 2006, p. 1-10. 14 Mentionnons deux colloques tenus en 2013: De l'archive au re-enactment: les enjeux de la re-présentation de la performance, Département des arts visuels de l'Université
Marina Abramovic apparaît comme la figure exemplaire de cette approche communément nommée le re-enactment.

À la suite des artistes, les spécialistes défendent aussi des conceptions diversifiées de la performance. L'approche ontologique de Peggy Phelan, par exemple, qui défend le caractère éphémère, unique et non reproductible de la pratique, est concurrencée par d'autres approches, comme celles d'Amelia Jones ou de Philip Auslander, qui reconnaissent à la documentation une légitimité et un pouvoir de transmission ${ }^{13}$. Depuis quelques années, ces spécialistes interrogent les enjeux de la transmission de la performance, de sa représentation, de sa recréation, de même que les rôles des objets, des archives et de la documentation au sein de ces actions ${ }^{14}$.

Dans la foulée de ces recherches, les musées interrogent également la valeur et le statut de l'archive et reconnaissent l'importance de la performance dans l'histoire de l'art récente. Plusieurs cherchent à développer des moyens d'intégrer la pratique à leurs collections. Par exemple, le Musée d'art moderne de New York (MoMA) met sur pied, au milieu des années 2000, le Department of Media and Performance Art, alors que la Tate Modern de Londres possède une équipe spécialement dédiée à la conservation du "time-based media art ", qui inclut la performance et les " travaux intangibles ${ }^{15}$. À plus petite échelle, déjà au tournant du siècle, les Fonds régionaux d'art contemporain Provence-Alpes-Côte d'Azur (FRAC P.A.C.A.) et FRAC Lorraine favorisent des politiques d'acquisition respectivement

de Strasbourg (avril) et Département d'histoire de l'art de l'Université Rennes 2 (septembre); et Entre documentation et recréation: mémoires et transmissions des œuvres performatives et chorégraphiques contemporaines, sous la direction d'Anne Bénichou, Fondation Jean-Pierre Perreault et Institut du patrimoine de l'UQAM, Montréal (mai).

15 MoMA, Media and Performance Art. <www.moma.org/ explore/collection/media> (consulté le 1er février 2013). À

la Tate Modern, un réseau de recherche intitulé Collecting the Performative a aussi vu le jour. Tate Modern. Conservation - Time-based Art. <www.tate.org.uk/about/our-work/conservation/time-based-media>; et Collecting the Performative. <www.tate.org.uk/about/projects/collecting-performative> (consultés le 1er février 2013). 
favorables aux « œuvres programmatiques, évolutives ou processuelles " ${ }^{16}$ et aux œuvres issues de la performance ou qui, plus largement, repoussent les " limites de la collection " ${ }^{17}$

\section{Déterminer la mise en forme d'une proposi- tion « muséalisable » et sa présentation}

Comme nous l'avons formulé en début d'article, l'un des enjeux de la conservation de la performance est la mise en forme d'un substitut qui détermine sa présence dans les collections muséales et qui respecte l'intégrité du travail de l'artiste. Par ailleurs, si les recherches sur la conservation de l'art contemporain montrent que le dialogue entre l'artiste et les professionnels des musées supportent les activités de conservation, de restauration et de présentation des œuvres, nous remarquons qu'un dialogue intervient en amont de l'entrée des pièces dans les collections, c'est-à-dire à l'étape décisive de la "sélection " du travail de l'artiste et de ses composantes ${ }^{18}$. Sans être exclusif à la pratique, ce dialogue, qui participe à la mise en forme de la proposition d'un artiste, est certainement plus fréquent à l'égard de la performance. Non seulement il arrive que les performances ou leurs objets associés ne se destinent pas a priori aux collections, ceux-ci ne sont pas nécessairement pensés pour subsister en tant que travail artistique une fois passé le moment fort de l'accomplissement de l'action. Pour Orlan comme pour Claudie Gagnon, la mise en forme d'une proposition muséalisable de leur performance respective répond d'abord à une invitation des institutions muséales intéressées par leur travail. Alors que le MNAM et Orlan composent un ensemble documentaire,

16 Acquises après de « longues discussions avec les artistes ", ces œuvres non traditionnelles du FRAC P.A.C.A., qui prennent la forme d'une " pièce générique", d'une "étape de projet " ou encore d'un " principe de discussion ", évoluent au rythme des interventions de l'artiste et des expositions. Elles représentent environ $20 \%$ des nouvelles pièces accueillies annuellement dans la collection, entre 2000 et 2004. MANGION, Éric (dir.). Prêts à prêter : acquisitions et rapport d'activités 2000-2004. FRAC P.A.C.A. Paris: Isthme; Marseille: FRAC P.A.C.A., 2005, p. 9.

17 FRAC LORRAINE. Bilan. Metz: FRAC Lorraine, 2009, p. 7. le MNBAQ et Claudie Gagnon imaginent une forme vivante, c'est-à-dire une version réitérable du travail performatif.

\section{L'approche documentaire: composer un ensemble témoin d'objets}

La performance intitulée Action ORLANCORPS. MesuRage du Centre Georges Pompidou appartient à une série de "MesuRages " qu'Orlan réalise, dans les années 1970 et au début des années 1980, dans les rues ou les places qu'elle visite et dans les musées où on l'invite. Ses performances consistent chaque fois à évaluer la longueur d'espaces choisis, non pas en mètres, mais en "Orlan-corps ", une unité de mesure personnelle qui équivaut à sa taille. Le titre des actions insiste sur le mot "Rage ", intensifié par une majuscule, qui est en fait celle de l'artiste qui " refuse de jouer le rôle qu'on veut lui imposer " ${ }^{19}$. L'action présentée au Centre Pompidou a lieu le 2 décembre 1977. Vêtue d'une robe blanche sans manches enfilée par-dessus ses vêtements, Orlan s'allonge au sol, marque le plancher d'une craie blanche et comptabilise le nombre d'Orlancorps parcourus. Sur un chevalet, elle écrit le titre de l'action, l'heure à laquelle elle commence et à laquelle elle se termine, ainsi que le nom de la ville où elle est présentée. Une fois le mesurage accompli, l'artiste retire sa robe, la plonge dans l'eau et la lave en public. Le rinçage terminé, elle verse le liquide sale dans de petites fioles avant de les sceller. À l'instar de ses autres actions, elle fait documenter sa performance par la photographie et la vidéo.

À la fin des années 2000, le MNAM souhaite faire l'acquisition de quelques œuvres d'Orlan dans le but d'enrichir sa collection et de préparer la tenue d'une grande exposition

18 La sélection d'un objet constitue, dans une conception séquentielle de l'intégration muséale d'une œuvre, la première étape du processus de muséalisation, la thésaurisation puis la présentation publique constituant les deux étapes subséquentes. DESVALLÉES, André et François MAIRESSE (dir.). "Muséalisation ". Dictionnaire encyclopédique de muséologie. Paris: Armand Colin, 2011, p. 251.

19 CROS, Caroline, Laurent LE BON et Vivian REHBERG. "1963-2003 Chronophotologie ». In. Bernard BLISTÈNE, Christine BUCI-GLUCKSMANN, Caroline CROS et al. Orlan. Paris: Flammarion, 2004, p. 32. 
exclusivement consacrée aux artistes femmes. Parmi les pièces convoitées, le choix du MNAM s'arrête sur deux performances réalisées en 1977, soit Le baiser de l'artiste et MesuRage du Centre Georges Pompidou. La deuxième, que nous venons de décrire, en plus d'appartenir à une série importante, fait partie de l'histoire des activités de l'institution muséale. Comme elle ne constitue pas un objet, le MNAM propose à l'artiste d'imaginer un ensemble d'éléments qui pourrait prendre la forme d'une proposition documentaire cohérente.

Aujourd'hui, cet ensemble est constitué de la "Robe des MesuRages " originale placée entre deux plexiglas, de la découpe d'un tirage photographique à l'effigie de l'artiste qui « brandit le liquide de rinçage ", de douze photographies de format moyen et de six petits tirages originaux noir et blanc issus de l'action, d'un constat (chevalet surmonté d'une fiole de verre comprenant le liquide et d'une photographie), d'une plaque commémorative en cuivre, d'une vidéo noir et blanc de l'action et du "Certificat pour l'entrée de l'ensemble dans les collections, comme les douze photographies qui sont des tirages récents.

Tous ces éléments font l'objet d'une sélection: d'autres sont en effet proposés, puis laissés de côté pour diverses raisons ${ }^{20}$. Au terme des discussions entre l'artiste et la conservatrice responsable de l'acquisition, les objets sont rassemblés pour former un "ensemble indissociable " intitulé Action ORLAN-CORPS. MesuRage du Centre Georges Pompidou ${ }^{21}$. Si certains éléments ont été exposés dans différents contextes au cours des trente années qui séparent la création de la performance et l'entrée dans les collections du MNAM de l'ensemble documentaire, le travail de catalogage scelle cet ensemble. Celui-ci constitue dès lors la manifestation légitime de la performance d'Orlan, reconnue comme telle par l'artiste et cautionnée par le musée qui l'accueille comme œuvre dans ses collections ${ }^{22}$.

L'ensemble est présenté pour la première fois dans le cadre de l'expositionelles@centrepompidou, qui ouvre ses portes en mai 2009. Sont répartis sur un même plan horizontal, au sol ou sur une cimaise du corridor central, la Robe, la reproduction grandeur nature d'Orlan qui bandit le liquide de rinçage, la vidéo, la plaque commémorative, ainsi que des photographies de moyen format. Dans une vitrine sont rassemblés les autres éléments, notamment les six petits tirages originaux et le certificat. Comme c'est souvent le cas pour les œuvres contemporaines, «l'accrochage [...] s'est fait en collaboration avec l'artiste "23. Bien qu'il constitue un groupe d'objets « indissociable ", l'ensemble n'est pas une installation. À la différence de

Robe des MesuRages de l'ensemble est un objet de discussion entre l'artiste et les professionnels qui réfléchissent à la constitution de l'ensemble. Cette interrogation apparaît au dossier d'œuvre.

22 Le logiciel de saisie de données du MNAM permet de nommer et d'octroyer un numéro d'inventaire à chacune des composantes, puis de les joindre sous un seul numéro d'ensemble. La fiche descriptive de l'ensemble, accessible en ligne depuis la base de données Videomuseum, présente de manière manifeste la composition et le caractère de cet ensemble. Videomuseum, Centre Pompidou <www.videomuseum.fr $>$. 23 Commentaire de la conservatrice, tiré d'un échange électronique le 23 octobre 2009. 
l'installation, pour laquelle la disposition des éléments est généralement invariable et constitutive de la proposition de l'artiste, l'ensemble documentaire autorise les variations en fonction du contexte, par exemple, ou de l'espace de présentation disponible ${ }^{24}$.

\section{L'approche vivante: adapter la perfor- mance de manière à engager la réitération} Deux modes d'existence, que propose déjà Gérard Genette dans L'œuvre de l'art, définissent la présence de la performance dans les collections muséales. "L'enregistrement " et "l'itération ", écrit Genette, sont les deux moyens « par lesquels une œuvre de performance peut, dans une certaine mesure, échapper à sa condition temporelle d'événement, à son caractère éphémère et singulatif $" 25$. L'enregistrement est une " reproduction visuelle et/ou sonore " de la performance, alors que l'itération est l'action de renouveler, de répéter la performance ${ }^{26}$. La première et ses déclinaisons, aussi première à avoir été exploitée par le musée, est la plus courante. Elle s'articule généralement autour de l'acquisition, de l'entreposage et de l'exposition de prolongements documentaires aux formes multiples. C'est la stratégie qu'empruntent le MNAM et Orlan pour intégrer dans la collection du musée la performance de l'artiste. La seconde, beaucoup plus marginale, exploite l'itération ou la réitération. Elle convoque la mise en forme d'instructions ou de protocoles de réitération qui visent à encadrer la reprise de la performance. C'est la stratégie qu'exploitent le MNBAQ et Claudie Gagnon pour intégrer à la collection le travail de performance de cette dernière.

Chez Gagnon, la pratique de la performance prend la forme du tableau vivant. Celui-ci emprunte à la tradition des cabarets et du living

24 La disposition des éléments peut varier. Mais la composition? Le terme " ensemble indissociable " est bien énoncé sur la fiche descriptive, ce qui signifie que les différents éléments ne constituent pas des objets autonomes. Toutefois, c'est bien six photographies sur douze qui composent l'accrochage au Centre Pompidou, en 2009. Il semble que la pièce Action ORLAN-CORPS. MesuRage du Centre Georges Pompidou puisse " exister " de manière intègre à travers l'exposition d'un ensemble incomplet.

25 GENETTE, Gérard. L'œuvre de l'art. Immanence et transcendance. Paris: Seuil, 1994, p. 76. display que Jennifer Fisher et Jim Drobnick définissent comme " un type précis de présentation qui est mis en scène et qui inclut des êtres vivants ${ }^{27}$. Son approche nécessairement hybride contraint les commentateurs à l'énumération de disciplines. Jérôme Delgado écrit que les tableaux vivants de Claudie Gagnon se situent " à la frontière de la peinture, du théâtre et de la performance ${ }^{28}$. Nathalie Côté affirme quant à elle qu'ils « ne sont pas strictement du théâtre, encore moins du mime [...] ni de la chanson, ni du cirque ${ }^{29}$. "C'est une fusion entre les arts visuels, le théâtre, la musique et la performance ", confirme l'artiste ${ }^{30}$.

En décembre 2008, dans le cadre d'une exposition collective intitulée C'est arrivé près de chez vous : l'art actuel à Québec, Claudie Gagnon présente, au MNBAQ, Dindons et limaces, un cabaret composé d'une vingtaine de tableaux vivants. Ces derniers apparaissent comme des pastiches de chefs-d'œuvre de l'histoire de l'art. Costumés et maquillés, huit comédiens et comédiennes incarnent dans des saynètes inquiétantes, touchantes ou comiques, des personnages tirés des Glaneuses de Jean-François Millet, du Cri d'Edward Munch et d'autres tableaux de Francis Goya, d'Edgar Degas ou de René Magritte. Des bruiteurs et des musiciens participent à cette manifestation.

Parce que la pratique du tableau vivant est représentative du travail de Claudie Gagnon, le MNBAQ choisit d'acquérir l'un de ces derniers. Avec le consentement de l'artiste, la conservatrice de l'art actuel fait le choix des Époux Arnolfini. À l'instar du plus célèbre tableau de Jan van Eyck, la proposition de Claudie Gagnon met en scène Monsieur et Madame Arnolfini. L'homme paraît côté jardin, habillé d'une tunique et d'un imposant

26 Id., p. 77.

27 FISHER, Jennifer et Jim DROBNICK. CounterPoses: re-concevoir le tableau vivant. Montréal: Oboro, 2002, p. 14. 28 DELGADO, Jérôme. "Cabaret muséal. Une exposition événement sur les arts faits à Québec est présentée dans la capitale ". Le Devoir, 12 décembre 2008, p. B1.

29 CÔTÉ, Nathalie. "La cour des miracles". Voir Québec, 5-11 octobre 2000, p. 17.

30 Ibid. 
chapeau, alors que sa promise se présente enceinte, côté cour, coiffée d'un foulard blanc et vêtue d'une robe verte. Entre les deux personnages se trouve un miroir bombé et, au-dessus des têtes, un chandelier. Si la première image que propose Gagnon reproduit le tableau du peintre flamand, la suite n'est que pure invention. D'une durée approximative de cinq minutes, le récit relate la poussée soudaine des contractions de la dame qui provoque le désarroi du futur père. Outre le jeu et l'allure clownesque des comédiens, c'est la mise en scène des anachronismes qui crée l'effet comique. La distribution réduite du tableau vivant et la ligne dramatique de son scénario lui garantissent une autonomie et une existence pertinente au-delà du cabaret. Ces deux aspects du tableau apparaissent comme des arguments en faveur de son entrée dans la collection.

Intégrer un tableau vivant dans la collection du MNBAQ, c'est se prévaloir du droit de le présenter à nouveau, dans des circonstances variables. C'est, par conséquent, obtenir le droit de réitération des Époux Arnolfini. Dans ce cas précis où le tableau n'est pas conçu pour la collection, c'est aussi déterminer les paramètres d'une proposition inédite et " muséalisable ». Par ailleurs, bien qu'il puisse être compris comme objet autonome, le tableau vivant demeure attaché aux autres tableaux du cabaret (le décor est le même, d'autres comédiens manipulent les accessoires pendant que jouent les époux). C'est donc, en premier lieu, un travail d'adaptation qui revient à la conservatrice et à l'artiste. À l'instar de la conservatrice du Centre Pompidou et d'Orlan, qui travaillent de concert au choix d'un ensemble documentaire, la conservatrice du MNBAQ et Claudie Gagnon choisissent ensemble, avec l'aide des collaborateurs du musée ou de l'entourage de l'artiste, la forme que prendra le tableau vivant dans la collection.

C'est grâce à des rencontres, des discussions, des échanges électroniques et la production de documents de travail qu'est élaborée progressivement une adaptation des Époux Arnolfini. Des aspects du tableau vivant sont d'abord identifiés comme éléments importants, puis redéfinis par l'artiste et la conservatrice. Ces aspects sont, par exemple, le texte ou la suite des gestes accomplis par les deux personnages et le mouvement des accessoires. Ils correspondent aussi à la scénographie, à l'éclairage, à l'habillage sonore, aux costumes et aux maquillages, à la distribution (les comédiens " originaux " ne seront pas toujours là), à la mise en scène et, enfin, au contexte de représentation dans un cadre muséal.

Pour illustrer notre propos, prenons l'exemple de la scénographie. Le cabaret Dindons et limaces est présenté sur une large scène montée temporairement dans une rotonde du MNBAQ. Le dispositif scénographique est pensé pour accueillir simultanément plusieurs comédiens, car certains tableaux requièrent des distributions importantes. Le dispositif comporte des rideaux de scène et une toile de fond qui est roulée sur elle-même après chacun des tableaux de manière à laisser apparaître un nouveau décor. La conservatrice et l'artiste doivent imaginer comment transformer ce large dispositif de représentation en un espace adapté aux Époux Arnolfini. Des interrogations nourrissent les discussions : vaut-il mieux exploiter les espaces plus ou moins clos dont dispose déjà le musée ou reconstituer de toutes pièces un petit théâtre pouvant être monté dans n'importe quelle salle de musée? Reconstituer ce petit théâtre, avec ses rideaux et ses toiles de fond, ou élaborer un dispositif complètement nouveau et plus léger ? Et quel tissu choisir pour les rideaux? Faut-il imaginer un système de poulies permettant de manipuler les rideaux ou les laisser simplement tomber sur les côtés? Et en ce qui concerne la toile de fond, doit-on récupérer une section de l'originale ou en fabriquer une seconde ${ }^{31}$ ?

À travers l'ébauche de diverses propositions, la conservatrice et l'artiste s'entendent pour faire construire un dispositif scénique autoportant 
et démontable. Sa structure peut recevoir un rideau qui peut être ouvert et fermé de manière à marquer clairement le début et la fin des représentations. Les rideaux sont taillés dans l'un des deux tissus que propose l'artiste et que choisit la conservatrice. La toile de fond n'est pas une section de l'originale, que l'artiste préfère conserver, mais une toile nouvelle, refaite selon ses indications.

Si la transformation de la scénographie est envisageable pour l'artiste et la conservatrice, d'autres aspects du tableau vivant doivent être préservés et éventuellement reproduits de la manière la plus semblable possible. Parmi ces aspects, il y a le scénario. Bien que la force du pastiche repose sur la présentation visuelle qui rappelle le tableau de Jan van Eyke, le scénario compose le cœur des Époux Arnolfini et définit ce que Genette identifie comme ses (ou une partie de ses) "propriétés constitutives " ${ }^{32}$. Alors que d'autres performances, comme celles d'Esther Ferrer, sont empreintes d'une grande liberté d'action qui autorise les variations d'une réitération à l'autre, le tableau vivant de Claudie Gagnon est réglé au quart de tour. La conservatrice et l'artiste tiennent à ce que soit reconduite avec précision chacune des actions.

À la demande de la conservatrice, l'artiste rédige donc a posteriori, c'est-à-dire après la présentation du cabaret et spécialement pour l'entrée du tableau vivant dans la collection du MNBAQ, les « Notes de jeu ». Ces notes constituent la liste, en ordre chronologique et avec mention du temps, de tout ce qui compose le tableau vivant: actions, expressions des visages, changements d'éclairage, effets sonores, manipulations d'accessoires, ouvertures et fermetures des rideaux ${ }^{33}$. Elles composent la partition des Époux Arnolfini ou, à tout le moins, la pièce maitresse du « script » de

32 GENETTE. L'œuvre de l'art. Immanence et transcendance., op. cit., p. 26.

33 Voici un extrait des "Notes de jeu ", dossier d'œuvre Les Époux Arnolfini, MNBAQ : "Les deux personnages attendent de prendre la pose pour le peintre. Attitude décontractée. / 0:10: Clavecin (hésitant) tout au long du tableau. / Les personnages s'animent: / l'Époux replace son vêtement / l'Épouse regarde au loin. / 0:46 à 1:14: Son du lustre qui descend du plafond. / Le lustre descend du plafond, lentement la performance. À l'instar de Francine Couture et Richard Gagnier, nous appelons " script " l'ensemble des instructions produites par l'artiste ou par l'institution collectionneuse qui ont pour but de déterminer « la version autorisée de l'œuvre qui sera présentée au public » ${ }^{34}$.

En somme, adapter le tableau vivant pour la collection du MNBAQ consiste à identifier les propriétés de la pièce originale jugées incontournables, comme le scénario, ou plutôt secondaires, comme la scénographie. Adapter le tableau vivant, c'est aussi déterminer de nouvelles propriétés qui deviennent, dès lors et pour l'avenir, les propriétés originales de la version adaptée du tableau, de sa version « muséalisée ». Imaginer un dispositif scénique inédit, en dessiner les plans, en construire les différentes parties et les entreposer dans les réserves, c'est déterminer à l'avance, avec l'artiste, un dispositif de présentation spécialement adapté au tableau, c'est-à-dire de la bonne taille et dans l'esprit du tableau et du travail de Claudie Gagnon. Produire un document qui consigne les "Notes de jeu " et fabriquer le dispositif scénique, c'est assurer au tableau une forme d'unité esthétique à travers les représentations éventuelles.

Mais si adapter le tableau vivant c'est préserver les propriétés constitutives du tableau dans le but de respecter l'intégrité de l'artiste et de son œuvre, c'est aussi reconnaître les responsabilités du musée en matière de conservation et s'assurer qu'elles puissent être honorées. Par exemple, c'est un argument visant le respect de l'esprit du tableau vivant couplé à un argument en faveur d'une conservation matérielle optimale des rideaux qui tranche en faveur du choix du tissu. Si le tissu préféré est plus coûteux à l'achat, il est aussi plus massif, plus théâtral et plus résistant. Pour la conservatrice,

au début puis en "perte de contrôle": trop bas, il accroche le chapeau de l'Époux. "

34 COUTURE, Francine et Richard GAGNIER. « Les valeurs de la documentation muséologique: entre l'intégrité et les usages de l'œuvre d'art ". In. BÉNICHOU, Anne (dir.). Ouvrir le document: enjeux et pratiques de la documentation dans les arts visuels contemporains. Dijon: Les presses du réel, 2010 , p. 42. 
le tissu choisi est non seulement plus près de la proposition originale de l'artiste d'un point de vue esthétique, il est aussi plus durable. Ultimement, ce que souhaite la conservatrice, c'est que puisse être présenté et prêté le tableau vivant. Un programme trop lourd pourrait freiner le projet d'une réitération au MNBAQ ou dans une autre institution.

Le travail d'adaptation est opéré au fil des questions, des transmissions d'informations et des négociations. L'artiste établit ses exigences et communique à la conservatrice des aspects de son processus créatif, son attachement à la toile de fond qu'elle désire faire reproduire à l'identique, par exemple, ou l'importance d'engager des comédiens qui ressemblent aux Époux Arnolfini de Jan van Eyck ou, à tout le moins, à ceux de son propre tableau. De son côté, la conservatrice partage sa compréhension de l'œuvre, souhaite conserver les costumes et les accessoires qu'elle juge indissociables du tableau vivant et communique ses obligations, qui portent notamment sur les limites des coûts de fabrication, d'entreposage et de réitération. Aux demandes de l'artiste et du musée, s'ajoutent les exigences et les droits des collaborateurs qui ont participé à la création des Époux Arnolfini dans le cadre du cabaret. Si la conservatrice et l'artiste jugent important, dans le cadre d'une adaptation, de tenir compte de l'éclairage, de la musique et plus généralement de l'habillage sonore, les droits des collaborateurs (qui sont aussi des créateurs) doivent être respectés ${ }^{35}$.

\section{Définir et communiquer le statut des composantes documentaires}

La performance est aujourd'hui présente dans les collections muséales. Si des ensembles documentaires, comme celui d'Orlan, ou des pièces que l'on peut réitérer, comme celle de Claudie Gagnon, gagnent progressivement

35 Ces questions sont d'une grande complexité. À ce jour, la trame sonore, qui vise à remplacer la musique et les sons produits en direct par les musiciens lors du cabaret, n'a pas pu être enregistrée. Comme le tableau vivant n'a pas encore connu sa première réitération. les collections, les œuvres qui représentent la performance sont généralement identifiées dans les catalogues comme des photographies, des films, des vidéos. Et c'est en effet ce qu'elles sont d'un point de vue matériel ou du point de vue du catalogage qui vise à identifier et à décrire, rappelons-le, des objets aux propriétés tangibles et mesurables. Bien que nous ne remettions pas en question ces règles qui assurent la normalisation des données et la connaissance des collections, il semble que ces photographies, films et vidéos appartiennent également à un autre monde, celui de la trace ou de l'objet associé à un événement antérieur significatif qui a eu lieu dans un autre contexte. En d'autres mots, ces pièces ne semblent appartenir ni tout à fait à la performance, ni tout à fait à la photographie (par exemple) ou semblent être à la fois de l'un et de l'autre. Les règles et les outils de documentation ne semblent pas parvenir à éclairer adéquatement cet entre-deux ${ }^{36}$.

Comme nous l'avons annoncé en début d'article, l'un des enjeux de la conservation de la performance est celui de la connaissance de ces différents éléments, de ceux qui composent l'ensemble documentaire d'Orlan, par exemple, de leur statut, ainsi que de la place qu'ils occupent au sein du travail performatif. En d'autres mots, que sont véritablement ces photographies, ces vidéos, ces objets, ces écrits? Sont-ils des enregistrements, des " reliques ", des travaux préparatoires? Ont-ils été simplement prélevés ou soigneusement mis en forme? Et cela, à l'époque de la réalisation de la performance ou dans un autre temps? Sont-ils des traces secondes dans l'esprit du travail de l'artiste ou, au contraire, les résultats attendus d'actions comprises comme des moyens de création? Ces questions sont importantes, car elles orientent le regard que l'on porte sur ces différents documents et, par extension, sur la performance.

36 Des documentalistes usent parfois d'astuces de catalogage et d'indexation pour évoquer la double appartenance de ces objets de musée particuliers. Pour des exemples, voir GIGUÈRE. Art contemporain et documentation: la muséalisation d'un corpus de pièces éphémères de type performance., op. cit., p. $102-110$. 
La question du statut des objets et des liens qu'ils entretiennent avec les performances est également pertinente à l'égard des actions qui s'inscrivent dans les collections sous la forme de réitérations éventuelles. Car, outre les " situations construites " de Tino Sehgal, rares sont les performances qui, dans les collections muséales, ne sont représentées par aucun objet de valeur ${ }^{37}$. De manière générale, ces objets sont reconnus comme des œuvres et comme des accessoires autour desquels, ou avec lesquels, la performance est réitérée. Admis dans la collection du FRAC P.A.C.A., destiné à être tantôt exposé, tantôt porté sur l'épaule par l'artiste ou par un médiateur dans le but de susciter des rencontres, l'âne de tissu de Stephen Wilk, par exemple, intitulé Âne bleu, est à la fois un objet de musée et un déclencheur de gestes performatifs ${ }^{38}$. Par ailleurs, il est également fréquent que des photographies ou des vidéos de nature documentaire accompagnent les performances dans les collections muséales.

En plus de participer à la définition d'un ensemble documentaire, Orlan et ses représentants, les conservateurs et les documentalistes du MNAM travaillent de concert à l'identification des différents éléments qui le composent. Des discussions entre les uns et les autres contribuent à déterminer la nature et le statut des objets, à spécifier, par exemple, que les images transmises constituent des " compléments d'information " et " non des œuvres " 39 . Des échanges électroniques, conservés au dossier d'œuvre, apparaissent comme des prolongements de ces discussions.

Aussi, Claudie Gagnon et la conservatrice du MNBAQ travaillent à l'identification du statut des éléments de la proposition muséalisée. L'entrée des Époux Arnolfini dans la collection du MNBAQ s'exprime à travers

37 Les « situations construites " de Tino Sehgal sont des actions essentiellement fondées sur la parole, le mouvement et l'échange d'idées. Voir, dans ce même numéro de Muséologies, l'article d'Isabelle Riendeau et l'entretien que nous ont accordé les conservatrices et l'archiviste des collections du Musée d'art contemporain de Montréal. 38 Voir la fiche descriptive d'Âne bleu. Videomuseum, FRAC P.A.C.A. <www.videomuseum.fr >. l'adaptation du tableau vivant, la production sur mesure de composantes comme le dispositif scénique, mais également l'acquisition d'objets de valeur et de documents. En effet, le MNBAQ acquiert les coiffes et les manteaux de Monsieur et Madame Arnolfini, de même que deux éléments de décor ou accessoires, le miroir et le chandelier. Ces costumes et ces objets sont authentiques, uniques et originaux : ils sont des créations ou des trouvailles de l'artiste et ils appartiennent déjà au cabaret. Mais ils sont aussi des accessoires de jeu. À la différence de l'âne de Stephen Wilk, reconnu à la fois comme œuvre, accessoire et objet de musée, les costumes, le miroir et le chandelier des Époux Arnolfini ne sont pas des objets de musée autonomes. Reconnaissant de concert l'appartenance ou la subordination des costumes et des accessoires aux Époux Arnolfini, la conservatrice choisit de ne pas les inscrire à l'inventaire, mais de les associer plus globalement au tableau vivant. À l'instar de l'ensemble documentaire d'Orlan, l'attribution d'un numéro d'inventaire, la production d'un dossier d'œuvre et le catalogage de la performance de Claudie Gagnon scellent le statut du tableau vivant, des costumes, du chandelier et du miroir, de la même manière qu'ils rejettent du domaine artistique la vidéo documentaire et les photographies prises lors du cabaret et de sa générale. Alors que le tableau vivant est énoncé comme œuvre, que les costumes, le chandelier et le miroir sont définis comme des composantes de l'œuvre, la vidéo et les photographies se voient octroyé le statut de document. L'entrée du tableau vivant dans la collection du MNBAQ engage d'ailleurs l'ajout d'un terme controversé dans les listes fermées des entrées « catégorie de l'objet » et " nom de l'objet " du catalogue, soit celui de " performance $"{ }^{40}$.

39 Il s'agit d'un commentaire de l'assistante d'Orlan, qui spécifie le statut de photographies envoyées à la conservation du MNAM dans le cadre du travail de sélection et de documentation de l'œuvre. Tiré d'un échange électronique conservé au dossier d'œuvre.

40 Une fiche descriptive sommaire des Époux Arnolfini, qui permet de constater ce choix de catalogage, est accessible dans la base de données Info-Muse. <http://infomuse.smq.qc.ca/ Infomuse/f_MasterLayout.cgi> (consulté le 1er février 2013). 
Il est attendu des professionnels qu'ils identifient la nature de ces d'objets et de ces documents, qu'ils éclairent leur histoire de production et leur statut, dans le but de communiquer cette connaissance aux regardeurs qui les considèrent. Dans le cadre des expositions, différentes stratégies peuvent être employées, dont, parmi les plus fréquentes, les textes d'accompagnement à la visite ou les cartels détaillés qui énoncent des éléments du contexte de la création de la performance. Dans les cas des performances discutées dans cet article, les titres des pièces, qui les représentent dans les collections, transmettent des informations pertinentes sur leur nature et leur association au travail performatif antérieur. Le titre complet de l'ensemble d'Orlan, tel qu'il est énoncé sur les cartels de l'exposition elles@centrepompidou, fait mention de la date de création de la performance: «Action ORLAN-CORPS. MesuRage du Centre Georges Pompidou, 2 décembre 1977. " Quant au titre complet du tableau vivant de Claudie Gagnon, tel qu'il est noté au catalogue, il explicite le caractère " adapté " de la proposition et réfère à son contexte de création original: «Les Époux Arnolfini, tiré du cabaret de tableaux vivants Dindons et limaces."

\section{Des traces de ces dialogues dans la documentation muséale}

La nature de l'art contemporain et la possibilité de s'entretenir directement avec les artistes ou leurs représentants définissent de nouveaux rôles et de nouvelles responsabilités pour ces derniers comme pour les professionnels des musées. Les territoires de la création et de la conservation, autrefois respectivement impartis aux premiers et aux deuxièmes, s'entrecroisent. Les connaissances, les savoir-faire et l'autorité des uns et des autres se trouvent partagés. Par ailleurs, artistes et professionnels sont perçus à la fois comme des partenaires et des négociateurs. Nous croyons, en effet, que leur dialogue est fait d'échanges plutôt que de simples transmissions d'informations de la part des artistes. Nous remarquons que les questions des conservateurs et des documentalistes, qui visent à répondre aux exigences muséales en matière de sélection, de catalogage, de conservation préventive ou d'exposition, amènent les artistes à se questionner sur leur travail, à déterminer a posteriori l'importance de certaines composantes, à départager ce qui appartient à une performance ou à une série de travaux.

Les recherches sur la conservation de l'art contemporain montrent que ces échanges et ces négociations se déploient sur une longue période, à travers un dialogue persistant, rythmé par les événements dans la vie de l'objet, ses sorties des réserves et ses restaurations. Si les deux cas étudiés dans cet article ne permettent que d'illustrer partiellement cette affirmation (parce qu'ils portent sur deux pièces nouvellement acquises), ils montrent que ce dialogue peut jouer un rôle crucial dans la mise en forme d'une proposition documentaire de la performance ou d'une proposition permettant de la représenter. Ce dialogue est également sollicité dans le travail de connaissance du statut des composantes des pièces ou des documents qui leur sont associés.

Nous avons retenu d'entrée de jeu le sens restreint du dialogue pour mettre en évidence le caractère direct, constructif et déterminant des échanges entre artistes et professionnels dans la conservation de l'art contemporain, et de manière plus spécifique de la performance. De la même manière que la performance se prolonge à travers des objets ou des documents qui prennent les formes de l'œuvre, de l'archive et du script, ces dialogues se trouvent, à un moment ou à un autre, inscrits dans une documentation muséale protéiforme qui vise à fixer les paramètres de l'œuvre et à nous renseigner sur ces différents aspects. Dans les dossiers d'œuvres, dans les communications diverses sur les œuvres, il y a des traces de ces dialogues. Il est ainsi possible d'imaginer un autre dialogue dans lequel un prochain conservateur prendra en compte ces discours dialogiques. 


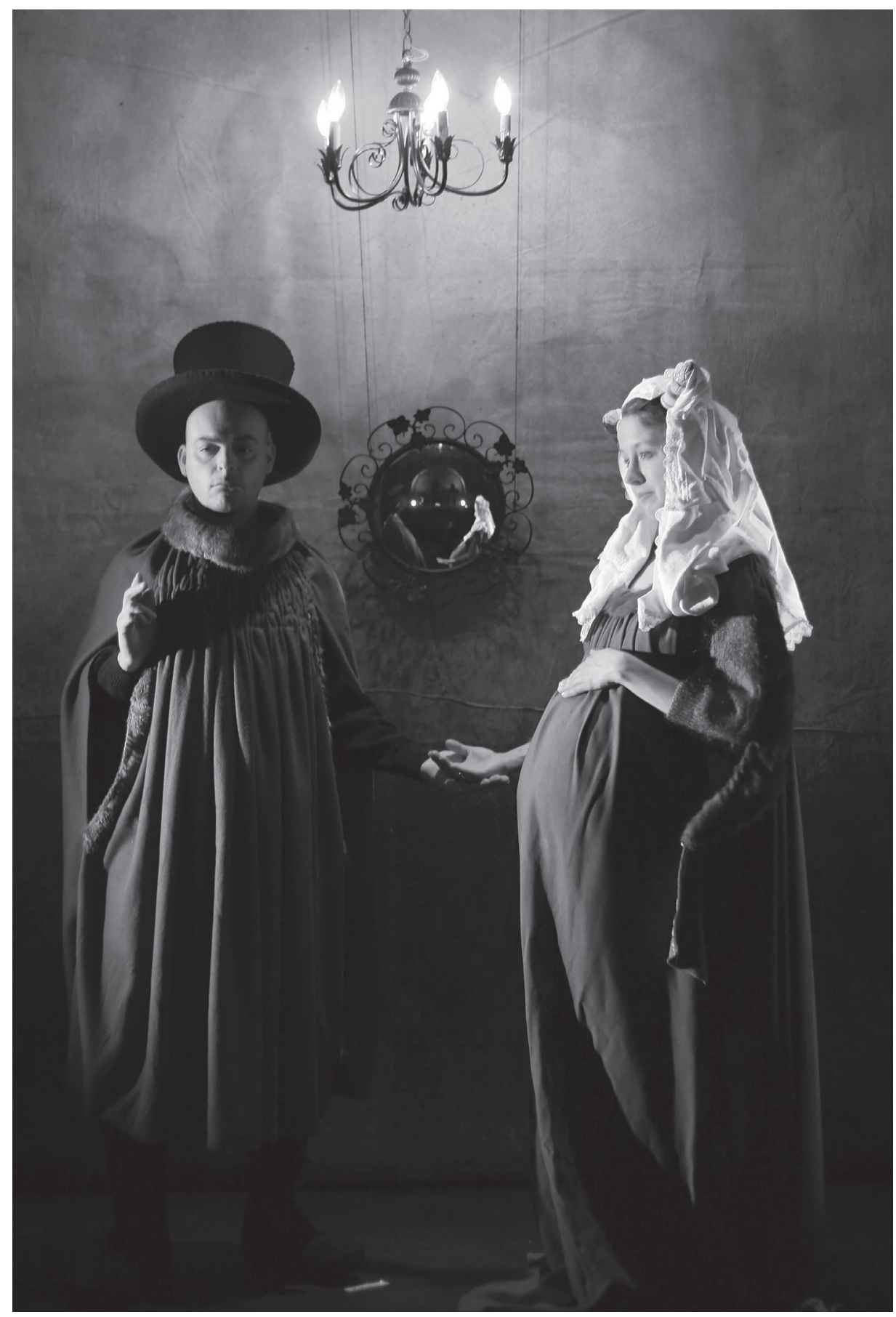

Claudie Gagnon

Les Époux Arnolfini, tiré du cabaret de tableaux vivants Dindons et limaces, 2008

Tableau vivant mettant en scène deux interprètes

Collection Musée national des beaux-arts du Québec (2009.89)

[Photo: Frédérick Lemieux] 


\section{Amélie Giguère, translated by Allana Carlyle}

For contemporary art, the use of fragile, unknown or ephemeral materials as well as the significant importance placed on the condition of the presentation of works, generates particular difficulties regarding the art's preservation, restoration and exhibition. Since the beginning of the 1990's, museum professionals, art historians and museologues have worked to resolve these difficulties and to understand the nature and the effects of these transformations on current museum practices. From these transformations, we see that professionals and current artists are being given new responsibilities. If, for example, curators are called to recreate an ephemeral work or to arrange elements of the installation in the space, artists are often called on to actively participate in the documentation of their work or to supervise their restorations. The fields of competence of these roles, once clearly defined, now intersect and create a space for discussion, collaboration and negotiation.

In the context of this shared responsibility, this article examines how through dialogue, curators, archivists, and artists prefer preservation, and in fact negotiate it's entry into museum collections and exhibits. Through the examination of two case studies, this article shows that the dialogue occurs at two strategic moments: Firstly during the shaping of a museum exhibition proposal and secondly in revealing the status of the various elements which make up the exhibition proposal. Based on this article review, the following questions are raised: How is the exhibition realized? How is it's renewal initiated? Which objects and documents are to be selected and defined? Are the objects collected, restored and documented works of art, archives or accessories? What is their relationship to the exhibit with which they are associated?

The first study focuses on the process of acquisition by the Musée national d'art modern in Paris at the end of the 2000s of a display by Orlan created in 1977 and entitled Action ORLAN-CORPS. MesuRage du Centre Georges Pompidou. The study demonstrates how the museum places a request to the artist, a negotiation and a sharing of information takes place, and the result is a documentary collection of the exhibition. The second study primarily examines the acquisition of a painting by Claudie Gagnon entitled Les Époux Arnolfini. This work was acquired by the Musée national des beaux-arts in Quebec also during the 2000s and was presented for the first time in 2008 . The study recounts the adaptation of a tableau vivant and its possible future rendering into living form, that is the form that was originally proposed by the artist.

In both cases, these articles demonstrate that it is dialogue which allows for the identification of the constituent properties of exhibitions that define their integrity. Also, that responses to questions raised through the work of musealization become apparent during the crossing of preservation and creation practices. In short, the articles show that the knowledge of the status of objects, archives and documents which 
replace or accompany exhibitions as well as the relationships they have with exhibitions, shed light on our relationship with the work of artists and consequently play a decisive role in the preservation of their work. 